\title{
PRÁTICAS DE ENFERMAGEM EM SAÚDE MENTAL NA ESTRATÉGIA DE SAÚDE DA FAMÍLIA: REVISÃO INTEGRATIVA*
}

Ana Monalisa Ferreira Merces' ${ }^{1}$, Bruna de Medeiros Leite Souza', Tatiane Lins da Silva², Thassia Thame de Moura Silva ${ }^{3}$, Ana Márcia Tenório de Souza Cavalcanti ${ }^{4}$

${ }^{1}$ Discente de Enfermagem. Universidade Federal de Pernambuco. Recife-PE-Brasil.

${ }^{2}$ Enfermeira. Mestranda em Enfermagem. Universidade Federal de Pernambuco. Recife-PE-Brasil.

${ }^{3}$ Enfermeira. Mestranda em Educação em Saúde. Professora da Universidade Federal de Pernambuco. Recife-PE-Brasil.

${ }^{4}$ Enfermeira. Doutora em Saúde da Criança e do Adolescente. Professora da Universidade Federal de Pernambuco. Recife-PE-Brasil.

RESUMO: Revisão Integrativa que objetivou identificar as práticas do enfermeiro em Saúde Mental na Estratégia de Saúde da Família. A busca dos dados foi realizada no período entre 29 de maio a 10 de junho de 2014, nas bases Literatura Latino-Americana e do Caribe em Ciências da Saúde, Literatura Internacional em Ciências da Saúde, Base de dados em enfermagem e na Biomedical Literature Citations and Abstracts. Foram encontrados nove artigos distribuidos em três categorias: Dificuldades e necessidade de capacitação em saúde mental, Principais práticas de enfermagem à pessoa com transtorno mental e Estratégia de Saúde da Família: Integrante da Rede de Saúde Mental. Conclui-se que os enfermeiros, atuantes neste cenário, posicionaram suas práticas em Saúde Mental baseadas no acolhimento, na renovação de receitas, na dispensação de medicação e no encaminhamento a serviços especializados. Entretanto, essas atitudes são reconhecidas como insuficientes na assistência em Saúde Mental. DESCRITORES: Saúde mental; Enfermagem; Estratégia de saúde da família.

\section{NURSING PRACTICES IN MENTAL HEALTH IN THE FAMILY HEALTH STRATEGY: AN INTEGRATIVE REVIEW}

\begin{abstract}
This integrative review aims to identify the nurse's practices in mental health in the Family Health Strategy. The search for data was undertaken in the period 29th May - 10th June 2014, in the following databases: the Latin American and Caribbean Center on Health Sciences Information, International Literature in Health Sciences, the Brazilian Nursing Database (BDENF) and in the Biomedical Literature Citations and Abstracts. A total of nine articles was found, distributed in three categories: Difficulties and the need for training in Mental Health, Main practices in nursing for the person with mental disorders and the Family Health Strategy: Member of the Mental Health Network. It is concluded that the nurses working in this scenario position their practices in Mental Health based in embracement, in renewing prescriptions, in dispensing drugs and in referral to specialized services. These attitudes, however, are recognized as insufficient in Mental Health care.
\end{abstract}

DESCRIPTORS: Mental Health; Nursing; Family Health Strategy.

\section{PRÁCTICAS DE ENFERMERÍA EN SALUD MENTAL EN LA ESTRATEGIA DE SALUD DE LA FAMILIA: REVISIÓN INTEGRATIVA}

RESUMEN: Revisión integrativa cuyo objetivo fue identificar las prácticas del enfermero en Salud Mental en la Estrategia de Salud de la Familia. La búsqueda de los datos fue realizada en el periodo entre 29 de mayo a 10 de junio de 2014, en las bases Literatura Latinoamericana y del Caribe en Ciencias de la Salud, Literatura Internacional en Ciencias de la Salud, Base de datos en enfermería y en la Biomedical Literature Citations and Abstracts. Fueron encontrados nueve artículos organizados en tres categorias: Dificultades y necesidad de capacitación en salud mental, Principales prácticas de enfermería a la persona con trastorno mental y Estrategia de Salud de la Familia: Integrante de la Red de Salud Mental. Se concluye que los enfermeros, actuantes en este área, posicionaron sus prácticas en Salud Mental basadas en acogimiento, en la renovación de recetas, en el almacenamiento de medicación y en el encaminamiento a servicios especializados. Sin embargo, esas actitudes son reconocidas como insuficientes en la asistencia en Salud Mental. DESCRIPTORES: Salud mental; Enfermería; Estrategia de salud de la familia.

*Artigo extraído do Trabalho de Conclusão de Curso intitulado: "Práticas em Saúde Mental do Enfermeiro Atuante na Estratégia de Saúde da Família". Universidade Federal de Pernambuco, 2014. 


\section{INTRODUÇÃO}

Nas últimas décadas houve crescente aumento da prevalência de transtornos mentais na população. Atualmente, segundo a Organização Pan-Americana de Saúde (OPAS) e a Organização Mundial de Saúde (OMS) cerca de 450 milhões de pessoas no mundo sofrem de transtornos mentais ou neurobiológicos ${ }^{(1)}$.

No Brasil, 3\% da população necessita de cuidados contínuos relacionados à Psiquiatria, ou seja, cerca de cinco milhões de brasileiros sofrem com algum transtorno mental severo e persistente. Esse percentual é acrescido pela demanda espontânea, ou seja, população que utiliza esses atendimentos eventualmente, o que totaliza 20 milhões de pessoas recorrendo a tratamentos adstritos à Saúde Mental(2).

Com o aumento gradativo das demandas de cuidado, às pessoas com transtornos mentais, surge a necessidade da elaboração de novos modelos de assistência. Com o movimento de Reforma Psiquiátrica no Brasil há novas experiências no tratamento em saúde mental, incluindo a substituição dos Hospitais Psiquiátricos por Centros de Atenção Psicossocial (CAPS) e a inclusão da Estratégia de Saúde da Família (ESF) nesta rede de atenção $^{(3)}$.

A inserção da ESF na rede tratamento da doença mental pauta-se na necessidade de tratamento e acompanhamento contínuos, que permita aos pacientes a ressignificação de sintomas e sofrimentos vividos, contando com a prática do acolhimento, usual no campo da saúde mental, além do desenvolvimento de atividades coletivas em geral, como caminhadas, palestras e atividades em grupo envolvendo a referida temática ${ }^{(4)}$.

As práticas de saúde mental na ESF devem estar alicerçadas no vínculo, na corresponsabilidade, no envolvimento e conhecimento do grupo familiar. É primordial que este serviço também pratique a metodologia da desconstrução manicomial, adotando modelo de base comunitária e territorial, no qual os usuários possam receber tratamento de forma humanizada ${ }^{(5)}$.

Entretanto, a atenção psicossocial no nível primário acaba se tornando um processo complexo, visto que essa abordagem assume o desafio de trabalhar com pessoas em sofrimento mental crônico e, muitas vezes, as equipes das
ESF expressam dificuldades de identificação e acompanhamento destes transtornos de forma comunitária $^{(6)}$.

Tendo em vista que a Atenção Básica constitui um plano privilegiado para o cuidado das necessidades em saúde mental, o enfermeiro, como atuante direto neste serviço, deve estar preparado para o atendimento aos portadores de transtornos mentais, auxiliando a reduzir os danos envolvidos e possível hospitalização do paciente ${ }^{(7)}$.

Neste contexto, o enfermeiro deve estar preparado a conduzir o cuidado ao usuário, família e comunidade, promovendo ações de inclusão do paciente nas diversas formas de organização do serviço, construindo novos espaços de reabilitação psicossocial ${ }^{(8)}$.

Diante da responsabilidade e as competências do enfermeiro, objetivou-se nesta pesquisa analisar produções científicas relacionadas às práticas em saúde mental do enfermeiro atuante na ESF. Neste escopo, buscou-se proporcionar um arcabouço de informações sobre essa temática, visando analisar esta atividade.

\section{MÉTODO}

Trata-se de uma Revisão Integrativa, método que reúne a produção científica relevante acerca de determinado tema, oferecendo acesso rápido e sintetizado aos resultados científicos de maior importância para a área estudada ${ }^{(9-10)}$.

As etapas para a elaboração desta revisão foram: a) identificação do problema; b) busca dos artigos na literatura; c) categorização dos estudos; d) avaliação da amostra; e) interpretação dos resultados; f) síntese dos artigos analisados ${ }^{(11)}$.

Como orientação da pesquisa utilizou-se a seguinte pergunta norteadora: Quais as práticas em Saúde Mental do Enfermeiro atuante na Estratégia de Saúde da Família? Para tanto, foram utilizados os seguintes critérios de inclusão: artigos originais, publicados em periódicos científicos disponíveis na íntegra, com acesso gratuito e que respondessem à pergunta norteadora; estudos disponíveis em inglês, português ou espanhol publicado entre janeiro de 2004 e junho de 2014.

Como critérios de exclusão, não foram selecionadas: pesquisas do tipo relatos de experiência, trabalhos de conclusão de curso de graduação, monografias de especialização, 
dissertações, teses, relatórios de pesquisa e resumos de eventos; artigos do tipo ensaio teórico, reflexões, revisões bibliográficas, cartas, resenhas, editoriais, livros, capítulos de livros, publicações governamentais, boletins informativos.

A busca dos artigos foi realizada entre 29 de maio a 10 de junho de 2014, percorrendo dois caminhos: 1) Na Biblioteca Virtual em Saúde (BVS) e 2) Banco de Bases de Dados de Coordenação de Aperfeiçoamento de Pessoal de Nível Superior (CAPES). As bases de dados incluídas nesta pesquisa foram: Literatura LatinoAmericana e do Caribe em Ciências da Saúde (LILACS), Literatura Internacional em Ciências da Saúde (MEDLINE), Base de dados em Enfermagem (BDENF) e Biomedical Literature Citations and Abstracts (PubMed).

As estratégias de busca foram guiadas pelos termos controlados, retirados dos Descritores em Ciências da Saúde (DeCS). Os termos selecionados foram: Saúde Mental (Mental Health); Estratégia Saúde da Família (Family Health Strategy) e Enfermagem (Nursing). Os mesmos foram cruzados entre si, em língua inglesa, partindo do descritor eleito como principal (Saúde Mental; Mental Health), como apresentado no Quadro 1.

O cruzamento dos descritores resultou em um total de 1.088 artigos, publicados nos últimos dez anos, em português, inglês e espanhol e com texto completo disponível. Para a seleção dos artigos, em um primeiro momento, foi realizada a leitura dos títulos resultando na seleção de 66 publicações e posteriormente feita a análise crítica dos resumos. Após esse segundo momento, obteve-se um total de nove artigos. O Quadro 2 apresenta a justificativa para a exclusão dos artigos.

Os estudos selecionados foram catalogados de acordo com um instrumento ${ }^{(10)}$ que contempla os seguintes itens: a) Identificação do artigo; b) Instituição sede do estudo; c) Tipo de publicação; d) Características metodológicas; e) Avaliação do rigor metodológico.

$\mathrm{Na}$ avaliação da qualidade dos estudos utilizou-se um instrumento previamente validado (Critical Appraisal Skills Programme - CASP), que oferece um meio de avaliar a qualidade metodológica dos estudos quantitativos e qualitativos, de forma objetiva, sistemática e de fácil entendimento. Este instrumento é composto por 10 itens pontuáveis e de acordo com a pontuação obtida, os artigos são classificados em duas categorias: A (06 a 10 pontos) estudos com boa qualidade metodológica e viés reduzido e, B (no mínimo 05 pontos) estudos com qualidade metodológica satisfatória, mas com potencial de viés aumentado ${ }^{(12)}$.

Quadro 1- Número de passos para cruzamentos dos descritores. Recife-PE-Brasil, 2014

\begin{tabular}{|l|c|}
\hline Passos & Cruzamento dos descritores \\
\hline $1^{\circ}$ & "Mental Health" AND "Family Health Strategy" \\
\hline $2^{\circ}$ & "Mental Health" AND "Nursing" \\
\hline $3^{\circ}$ & "Mental Health" AND "Family Health Strategy" AND "Nursing" \\
\hline
\end{tabular}

Quadro 2 - Critérios para exclusão dos artigos segundo bases de dados. Recife-PE-Brasil, 2014

\begin{tabular}{|l|c|c|c|c|c|}
\hline Critério & BDENF & MEDLINE & LILACS & PubMed & Total \\
\hline Total localizado & 48 & 825 & 115 & 100 & 1.088 \\
\hline $\begin{array}{l}\text { Não se enquadra à temática do } \\
\text { estudo }\end{array}$ & 26 & 800 & 74 & 91 & 991 \\
\hline Artigos repetidos & 4 & 8 & 21 & 2 & 35 \\
\hline $\begin{array}{l}\text { Não corresponde à questão } \\
\text { norteadora }\end{array}$ & 13 & 17 & 16 & 7 & 53 \\
\hline Total Selecionado & 5 & 0 & 4 & 0 & 9 \\
\hline
\end{tabular}




\section{RESULTADOS}

Os nove artigos que constituem a amostra foram lidos na íntegra, catalogados e avaliados quanto ao rigor metodológico. Destes, oito artigos obtiveram conceito A e um conceito $B$, segundo a análise CASP(12). A seguir, o Quadro 3 sintetiza a amostra de forma catalogada.

Verificou-se que a maioria dos estudos analisados era de abordagem qualitativa. Todos foram publicados em língua portuguesa. Dentre os trabalhos os mais encontrados eram das regiões nordeste e sudeste Do Brasil.

O Quadro 4 apresenta um resumo dos aspectos analisados, proporcionando a comparação e melhor compreensão dos seus objetivos, tipo de estudo, principais resultados e conclusões.

Quadro 3 - Código dos artigos segundo título, autor, base de dados, periódico e ano de publicação da amostra. Recife-PE-Brasil, 2014

\begin{tabular}{|c|c|c|c|c|c|}
\hline Código & Titulo do artigo & Autor & Base & Periódico & Ano \\
\hline 1 & $\begin{array}{l}\text { Saúde mental e } \\
\text { enfermagem na } \\
\text { estratégia saúde da } \\
\text { família: como estão } \\
\text { atuando os enfermeiros? }\end{array}$ & $\begin{array}{l}\text { Ribeiro LM, } \\
\text { M e d e i r o s } \\
\text { SM, } \\
\text { Albuquerque } \\
\text { JS, } \\
\text { Ferna nd e s } \\
\text { SMBA. }\end{array}$ & BDENF & $\begin{array}{l}\text { Revista da } \\
\text { Escola de } \\
\text { Enfermagem } \\
\text { da USP }\end{array}$ & 2010 \\
\hline 2 & $\begin{array}{l}\text { Acolhimento e saúde } \\
\text { m e ntal: des a fi o } \\
\text { profissional na Estratégia } \\
\text { Saúde da Família. }\end{array}$ & $\begin{array}{l}\text { Sucigan DHI, } \\
\text { Toledo VP, } \\
\text { Garcia APRF. }\end{array}$ & BDENF & $\begin{array}{l}\text { Revista de } \\
\text { Rede de } \\
\text { Enfermagem } \\
\text { doNordeste } \\
\text { (Rene) }\end{array}$ & 2012 \\
\hline 3 & $\begin{array}{l}\text { O t r a b a l ho de } \\
\text { enfermagem em saúde } \\
\text { mental na Estratégia de } \\
\text { Saúde da Família. }\end{array}$ & $\begin{array}{l}\text { Oliveira FB, } \\
\text { Silva JCC, } \\
\text { Silva VHF, } \\
\text { C a r t a x o } \\
\text { CKA. } \\
\end{array}$ & BDENF & $\begin{array}{l}\text { Revista de } \\
\text { Rede de } \\
\text { Enfermagem } \\
\text { do Nordeste } \\
\text { (Rene) }\end{array}$ & 2011 \\
\hline 4 & $\begin{array}{l}\text { A percepção dos } \\
\text { enfermeiros de uma } \\
\text { universidade pública } \\
\text { do Piauí sobre saúde } \\
\text { mental na Estratégia } \\
\text { Saúde da Família. }\end{array}$ & $\begin{array}{l}\text { Moura MC, } \\
\text { Bernardes SB, } \\
\text { Rocha MLF. }\end{array}$ & BDENF & $\begin{array}{l}\text { Revista } \\
\text { Enfermagem } \\
\text { em Foco }\end{array}$ & 2010 \\
\hline 5 & $\begin{array}{l}\text { A saúde mental no } \\
\text { Programa de Saúde da } \\
\text { Família. }\end{array}$ & $\begin{array}{l}\text { Souza AJF, } \\
\text { Matias GN, } \\
\text { Gomes KFA, } \\
\text { P a r e n t e } \\
\text { ACM. }\end{array}$ & BDENF & $\begin{array}{l}\text { Revista } \\
\text { Brasileira de } \\
\text { Enfermagem }\end{array}$ & 2007 \\
\hline 6 & $\begin{array}{l}\text { A s s i s tê n c i a d e } \\
\text { enfermagem às pessoas } \\
\text { com transtornos mentais } \\
\text { e às famílias na atenção } \\
\text { básica. }\end{array}$ & $\begin{array}{l}\text { W a i d } \mathrm{m} \text { a } \mathrm{n} \\
\text { MAP, } \\
\text { Marcon SS, } \\
\text { Pandini A, } \\
\text { Bessa JB, } \\
\text { Paiano M. }\end{array}$ & LILACS & $\begin{array}{l}\text { Acta } \\
\text { Paulista de } \\
\text { Enfermagem }\end{array}$ & 2012 \\
\hline
\end{tabular}




\begin{tabular}{|c|c|c|c|c|c|}
\hline 7 & $\begin{array}{l}\text { As estratégias dos } \\
\text { enfermeiros para o } \\
\text { cuidado em saúde } \\
\text { mental no programa } \\
\text { saúde da família. }\end{array}$ & $\begin{array}{l}\text { Amarante AL, } \\
\text { Lepre AS, } \\
\text { Gomes JLD, } \\
\text { Pereira AV, } \\
\text { Dutra VFD. }\end{array}$ & LILACS & $\begin{array}{l}\text { Texto } \\
\text { Contexto } \\
\text { Enfermagem }\end{array}$ & 2011 \\
\hline 8 & $\begin{array}{l}\text { Perfil dos enfermeiros } \\
\text { da estratégia saúde da } \\
\text { família e suas habilidades } \\
\text { para atuar na saúde } \\
\text { mental. }\end{array}$ & $\begin{array}{l}\text { Gonçalves } \\
\text { RMDA, } \\
\text { Pedrosa LAK }\end{array}$ & LILACS & $\begin{array}{l}\text { Ciência } \\
\text { Cuidado e } \\
\text { Saúde }\end{array}$ & 2009 \\
\hline 9 & $\begin{array}{l}\text { O enfermeiro e as ações } \\
\text { de saúde mental nas } \\
\text { unidades básicas de } \\
\text { saúde. }\end{array}$ & $\begin{array}{l}\text { Caixeta CC, } \\
\text { Moreno V. }\end{array}$ & LILACS & $\begin{array}{l}\text { Revista } \\
\text { Eletrônica de } \\
\text { Enfermagem }\end{array}$ & 2008 \\
\hline
\end{tabular}

Quadro 4 - Código do artigo segundo tipo, objetivo, resultados e conclusões. Recife-PE-Brasil, 2014

\begin{tabular}{|c|c|c|c|c|}
\hline Código & Tipo de estudo & Objetivos & Resultados & $\begin{array}{l}\text { Conclusões e/ou } \\
\text { recomendações }\end{array}$ \\
\hline 1 & $\begin{array}{l}\text { Analítico, com } \\
\text { abordagem } \\
\text { qualitativa. }\end{array}$ & $\begin{array}{l}\text { Descrever as } \\
\text { atividades voltadas } \\
\text { para a atenção } \\
\text { ao portador de } \\
\text { transtorno mental } \\
\text { na ESF. }\end{array}$ & $\begin{array}{l}\text { Não existem } \\
\text { atividades voltadas } \\
\text { para o portador } \\
\text { de transtorno } \\
\text { mental e há falta } \\
\text { de capacitação dos } \\
\text { enfermeiros. }\end{array}$ & $\begin{array}{l}\text { É preciso haver } \\
\text { qualificação } \\
\text { profissional. }\end{array}$ \\
\hline 2 & $\begin{array}{l}\text { Exploratório- } \\
\text { descritivo, com } \\
\text { abordagem } \\
\text { qualitativa. }\end{array}$ & $\begin{array}{l}\text { Compreender } \\
\text { como os } \\
\text { enfermeiros da } \\
\text { ESF realizam o } \\
\text { acolhimento dos } \\
\text { pacientes de saúde } \\
\text { mental. }\end{array}$ & $\begin{array}{l}\text { O acolhimento se } \\
\text { caracterizou pelo } \\
\text { encaminhamento } \\
\text { a serviços } \\
\text { especializados. }\end{array}$ & $\begin{array}{l}\text { Características: } \\
\text { entrega de receitas, } \\
\text { orientação sobre } \\
\text { medicamentos e } \\
\text { encaminhamento a } \\
\text { outros serviços. }\end{array}$ \\
\hline 3 & $\begin{array}{l}\text { Exploratório- } \\
\text { descritivo, com } \\
\text { abordagem } \\
\text { qualitativa. }\end{array}$ & $\begin{array}{l}\text { Identificar e } \\
\text { avaliar os cuidados } \\
\text { oferecidos pelos } \\
\text { enfermeiros da } \\
\text { ESF frente às } \\
\text { necessidades de } \\
\text { saúde mental. }\end{array}$ & $\begin{array}{l}\text { Práticas em } \\
\text { saúde mental: } \\
\text { visita domiciliar, } \\
\text { encaminhamento } \\
\text { aos serviços } \\
\text { especializados } \\
\text { e orientação } \\
\text { aos Agentes } \\
\text { Comunitários de } \\
\text { Saúde (ACS), para a } \\
\text { realização de busca } \\
\text { ativa. Concepções } \\
\text { baseadas no modelo } \\
\text { biológico. }\end{array}$ & $\begin{array}{l}\text { A falta de interação } \\
\text { entre a ESF e } \\
\text { os serviços de } \\
\text { referência em saúde } \\
\text { mental é vista } \\
\text { como um grande } \\
\text { problema. }\end{array}$ \\
\hline
\end{tabular}




\begin{tabular}{|c|c|c|c|c|}
\hline 4 & $\begin{array}{l}\text { Abordagem } \\
\text { qualitativa }\end{array}$ & $\begin{array}{l}\text { Identificar as } \\
\text { percepções dos } \\
\text { enfermeiros que } \\
\text { atuam na docência } \\
\text { sobre as ações } \\
\text { desenvolvidas na } \\
\text { ESF para indivíduos } \\
\text { com transtorno } \\
\text { mental. }\end{array}$ & $\begin{array}{l}\text { Evidenciou-se que } \\
\text { os enfermeiros da } \\
\text { ESF atuam de forma } \\
\text { restrita no cenário da } \\
\text { saúde mental. }\end{array}$ & \begin{tabular}{|l} 
A falta de \\
capacitação \\
pode determinar \\
dificuldades para \\
desenvolver ações.
\end{tabular} \\
\hline 5 & $\begin{array}{l}\text { Quantitativo } \\
\text { descritivo. }\end{array}$ & $\begin{array}{l}\text { Identificar a } \\
\text { formação e } \\
\text { as ações do } \\
\text { enfermeiro em } \\
\text { Saúde Mental na } \\
\text { ESF. }\end{array}$ & $\begin{array}{l}\text { A maioria não possui } \\
\text { experiência e suas } \\
\text { ações de saúde } \\
\text { mental acontecem de } \\
\text { forma esporádica. }\end{array}$ & $\begin{array}{l}\text { Políticas públicas } \\
\text { voltadas a esta } \\
\text { área devem ser } \\
\text { rediscutidas para } \\
\text { direcionar caminhos } \\
\text { à reforma nas ações e } \\
\text { nos serviços. }\end{array}$ \\
\hline 6 & $\begin{array}{l}\text { Exploratório- } \\
\text { descritivo, com } \\
\text { abordagem } \\
\text { qualitativa. }\end{array}$ & $\begin{array}{l}\text { Conhecer como } \\
\text { os enfermeiros } \\
\text { que atuam na ESF } \\
\text { percebem sua } \\
\text { capacitação, para } \\
\text { assistir a pessoa com } \\
\text { transtorno mental e } \\
\text { sua família. }\end{array}$ & $\begin{array}{l}\text { Os enfermeiros } \\
\text { não se sentem } \\
\text { capacitados e as } \\
\text { atividades de saúde } \\
\text { mental se restringem } \\
\text { às ações da própria } \\
\text { rotina da UBS. }\end{array}$ & $\begin{array}{l}\text { Não existem } \\
\text { atividades voltadas à } \\
\text { promoção da saúde } \\
\text { mental. }\end{array}$ \\
\hline 7 & $\begin{array}{l}\text { Exploratório- } \\
\text { descritivo, com } \\
\text { abordagem } \\
\text { qualitativa. }\end{array}$ & $\begin{array}{l}\text { Descrever as } \\
\text { estratégias } \\
\text { utilizadas pelos } \\
\text { "enfermeiros da } \\
\text { família" no cuidado } \\
\text { aos pacientes em } \\
\text { sofrimento psíquico. }\end{array}$ & $\begin{array}{l}\text { Há dificuldade } \\
\text { de identificar a } \\
\text { atenção à saúde } \\
\text { dos portadores de } \\
\text { sofrimento psíquico, } \\
\text { como parte da } \\
\text { operacionalização } \\
\text { das ações no } \\
\text { cotidiano da ESF. }\end{array}$ & $\begin{array}{l}\text { Ações específicas } \\
\text { não são planejadas, } \\
\text { mas existem } \\
\text { estratégias indiretas } \\
\text { de cuidado às } \\
\text { pessoas em } \\
\text { sofrimento. }\end{array}$ \\
\hline 8 & $\begin{array}{l}\text { Quantitativo de } \\
\text { caráter descritivo e } \\
\text { transversal }\end{array}$ & $\begin{array}{l}\text { Identificar } \\
\text { habilidades dos } \\
\text { enfermeiros da ESF } \\
\text { para atuar na saúde } \\
\text { mental } \\
\end{array}$ & $\begin{array}{l}\text { A maior parcela de } \\
\text { enfermeiros se sente } \\
\text { despreparado para } \\
\text { lidar com a saúde } \\
\text { mental na ESF. }\end{array}$ & $\begin{array}{l}\text { É necessário o } \\
\text { estabelecimento de } \\
\text { interface das ações } \\
\text { de saúde mental e a } \\
\text { ESF. } \\
\end{array}$ \\
\hline 9 & $\begin{array}{l}\text { Qualitativo } \\
\text { descritivo/ } \\
\text { exploratório }\end{array}$ & $\begin{array}{l}\text { Aprender como } \\
\text { os enfermeiros de } \\
\text { unidades básicas } \\
\text { reconhecem as } \\
\text { ações de saúde } \\
\text { mental. }\end{array}$ & $\begin{array}{l}\text { Não existem ações } \\
\text { programáticas } \\
\text { dirigidas à saúde } \\
\text { mental, sendo } \\
\text { vistas como ações } \\
\text { de saúde mental } \\
\text { apenas o controle e } \\
\text { a orientações sobre } \\
\text { o uso da medicação } \\
\text { psiquiátrica. }\end{array}$ & $\begin{array}{l}\text { É necessário } \\
\text { capacitação e é } \\
\text { fundamental o } \\
\text { estabelecimento de } \\
\text { uma interface com } \\
\text { as ações dirigidas à } \\
\text { saúde mental. }\end{array}$ \\
\hline
\end{tabular}




\section{DISCUSSÃO}

A maioria dos artigos selecionados apresentou boa qualidade metodológica e viés reduzido, segundo a análise CASP, oferecendo credibilidade aos resultados alcançados. Todos os artigos encontraram-se em língua portuguesa o que pode ser explicado pelo fato de a ESF ser um modelo de atenção à saúde em prática no Brasil ${ }^{(13)}$.

Houve grande recorrência de pesquisas do tipo qualitativa. Esta abordagem corresponde a um domínio das relações e se trabalha o universo dos significados ${ }^{(14)}$, evidenciando que os autores buscaram compreender o modo como os enfermeiros enxergam o próprio trabalho em saúde mental dentro da ESF.

Também, emerge desta pesquisa a existência de poucos estudos sobre as atividades de saúde mental do enfermeiro na ESF, o que traz a necessidade de pesquisas de caráter exploratório, que buscam um maior aprofundamento teórico para se conhecer melhor um fenômeno ${ }^{(15)}$.

Os dados relevantes encontrados na amostra foram agrupados em três categorias, na busca de sintetizar e articular o conhecimento obtido, como abordado a seguir:

\section{Dificuldades e necessidade de capacitação em saúde mental dos enfermeiros atuantes na ESF}

Os enfermeiros, sujeitos das pesquisas analisadas, relataram falta de preparo, treinamento e capacitação para trabalhar com a demanda de saúde mental, o que pode ser reflexo de limitações individuais, da pouca experiência na área, ser iniciante na ESF e deficiências na formação acadêmica. Esses fatores fazem com que encontrem maiores dificuldades frente às diferentes e desafiadoras situações no cuidado à pessoa com transtorno mental ${ }^{(16-18)}$.

Os artigos descrevem que os enfermeiros não recebem incentivos da gestão em cursos de capacitação em Saúde Mental|(16-19), e que a parcela que busca essa formação, por conta própria, é muito pequena ${ }^{(20-21)}$. Diante desse contexto, pode ser afirmado que a formação e qualificação em atenção à pessoa com doença mental crônica, por profissionais de enfermagem que atuam na ESF, pode contribuir para a melhoria da qualidade da assistência à saúde mental(22).

\section{Principais práticas de enfermagem à pessoa com transtorno mental}

Estudos aqui analisados mostram que após três décadas do início da Reforma Psiquiátrica foram poucas as mudanças relacionadas à prática dos profissionais de saúde, que prestam assistência à pessoa com doença mental. Estas estão ainda centrada no atendimento individualizado e na medicalização, com ênfase na doença e não no sofrimento do sujeito ${ }^{(21)}$. Muitos profissionais não acompanharam as mudanças que o movimento trouxe e, portanto, não estão adequadas as novas práticas inseridas no cenário do cuidado à saúde mental ${ }^{(23)}$.

$\mathrm{Na}$ ESF os enfermeiros dos estudos pesquisados referem à inexistência de uma ação específica para saúde mental, de modo que a assistência à saúde mental está dentro das atividades rotineiras da unidade, sendo reconhecidas como mais importantes neste âmbito o encaminhamento aos serviços especializados, o aconselhamento realizado diante da crise $^{(21)}$ e a transcrição de medicação ${ }^{(23)}$.

Existe uma tendência terapêutica voltada ao modelo biológico que privilegia a medicalização do doente, havendo assim a transcrição de medicação sem a devida avaliação clínica, levando ao consumo abusivo de psicofármacos gerando dependência ${ }^{(23)}$. Isso acontece devido à falta de preparo dos profissionais da ESF, limitando o cuidado oferecido à pessoa com transtorno mental e a prática cotidiana destes serviços ao conhecimento da psiquiatria tradicional ${ }^{(21)}$.

Outra prática constante no atendimento em saúde mental na ESF é o encaminhamento como forma de acolhimento. Evidenciou-se que a função da enfermagem no acolhimento baseia-se em escutar a queixa e avaliar a necessidade do paciente ser atendido pelo médico da unidade ${ }^{(16)}$.

A Política Nacional de Saúde Mental propõe que as práticas nesse campo do saber, devem ser substitutivas ao modelo tradicional, produtor da psiquiatriazação do sujeito $^{(24)}$. É importante a inserção em grupos e redes sociais de suporte e realização de intervenções psicossociais a exemplo da terapia comunitária ${ }^{(25)}$. 
Estratégia de Saúde da Família: Integrante da Rede de Saúde Mental

Um dos estudos desta pesquisa mostrou a dificuldade do enfermeiro, em saber o que fazer durante $\mathrm{o}$ atendimento a uma pessoa com transtorno mental na ESF. Este fato acaba resultando no encaminhamento destes pacientes para um serviço especializado, prática vista como única alternativa para solucionar esse impasse ${ }^{(16)}$.

Fato este reforçado em outras pesquisas revelando que os serviços para encaminhamento em psiquiatria são frequentemente usados como alternativa de resolubilidade do problema em saúde mental, incluindo o Hospital Psiquiátrico e o ambulatório(20). Este situação quebra a integração da ESF à rede de Saúde Mental, a qual foi inserida após a Reforma Psiquiátrica ${ }^{(17)}$. O problema que surge por falta articulação entre a ESF com esses serviços especializados ${ }^{(26)}$.

As atividades do matriciamento em Saúde Mental disponibilizam a ampliação de acesso e diversificação da atenção à saúde direcionada para a integralidade, com o objetivo de minimizar a prática de encaminhamentos e elevar a resolubilidade assistencial, fazendo com que o profissional, dentre eles o enfermeiro, reconheça as condições sociais da demanda e possivelmente supere as condutas medicamentalizadas de saúde ${ }^{(27)}$

O momento atual exige a conjugação de esforços, no sentido de viabilizar novas formas de pensar e agir em relação ao processo saúdedoença mental, possibilitando a produção de uma rede de espaços de acolhimento e vínculos no território de atuação da ESF. A interlocução entre as redes especializadas com a ESF amplia o potencial desse dispositivo de atenção para novas formas de cuidado, para gradativamente diminuir a demanda de encaminhamento desses usuários ${ }^{(24)}$.

\section{CONCLUSÕES}

Foi evidenciado nos estudos analisados, que os enfermeiros atuantes na ESF, posicionam suas práticas em Saúde Mental, baseadas no acolhimento, na renovação de receitas, na dispensação de medicação e no encaminhamento desses casos a outros serviços especializados.
Entretanto, essas atitudes são reconhecidas por esses profissionais, como insuficientes diante o tratamento destes transtornos.

Os estudos mostraram a baixa incidência de atividades de promoção à saúde, elaborada pelo enfermeiro da ESF, voltadas aos transtornos mentais. Esta condição corrobora com a não identificação deste profissional, no pouco reconhecimento da ESF como parte da Rede de Atenção em Saúde Mental, além dos relatos de falta de habilidade e de capacitação para lidar com a questão.

É necessário que haja desde a graduação preocupação maior com este aspecto da formação, preparando os futuros enfermeiros para melhor atuação em saúde mental nos diversos cenários do cuidar. Evidencia-se também, a necessidade da contínua capacitação e atualização dos profissionais inseridos na ESF, visando maior interação com a população e a implementação de técnicas mais eficazes envolvendo a Saúde Mental.

\section{REFERÊNCIAS}

1. Reis LN, Pereira SS, Cardoso L, Gherardi-Donato, ECS. Transtornos mentais orgânicos em um ambulatório de Saúde Mental brasileiro. Rev. port. enferm. saúde mental. 2013;1(09):48-53.

2. Oliveira SA. Prevenção em saúde mental no Brasil na perspectiva da literatura e de especialistas da área [dissertação] Brasília (DF): Universidade de Brasília; 2012.

3. Presotto RF. Participação de usuários de serviços de saúde mental em pesquisas: um olhar a partir dos conceitos de empowerment e recover. [dissertação]. Campinas (SP): Unicamp; 2013.

4. Silva MCF, Furegato ARF, Júnior MLC. Depressão: pontos de vista e conhecimento de enfermeiros da rede básica de saúde. Rev Latino-Am. Enfermagem. 2003;11(1):7-13.

5. Silveira MFA, Felix LGF, Araújo DVA, Silva ICS. Acolhimento no programa saúde da família: um caminho para humanização da atenção à saúde. Cogitare enferm. 2004;9(1):71-8.

6. Lucchese R, Oliveira AGB, Conciani ME, Marcon SR. Saúde mental no programa saúde da família: caminhos e impasses de uma trajetória necessária. Cad. saude publica. 2009;25(9):2033-42.

7. Neves HG, Lucchese R, Munari DB. Saúde mental 
na atenção primária: necessária constituição de competências. Rev. bras. enferm. 2010;63(4):666-70.

8. Büchele F, Laurindo DLP, Borges VF, Coelho EBS. A interface da saúde mental na atenção básica. Cogitare enferm. 2006;11(3):226-33.

9. Mendes KDS, Silveira RCCP, Galvão CM. Revisão integrativa: método de pesquisa para a incorporação de evidências na saúde e na enfermagem. Texto Contexto Enferm. 2008;17(4):758-64.

10. Souza MT, Silva MD, Carvalho R. Revisão integrativa: o que é e como fazer. Einstein. 2010;8(1):102-6.

11. Pompeo DA, Rossi LA, Galvão CM. Revisão integrativa: etapa inicial do processo de validação de diagnóstico de enfermagem. Acta Paul. Enferm. 2009;22(4):434-8.

12. University of Kent Templeman Library. [Internet]. Critical appraisal skills Programme (CASP). Canterbury (KE): University of Kent; [acesso em 25 jun 2014]. Disponível: http://library.kent.ac.uk/library/info/ subjects/healthinfo/critapprais.html

13. Escorel S, Giovanella L, Mendonça MHM, Senna MCM. O Programa de saúde da família e a construção de um novo modelo para a atenção básica no Brasil. Rev Panam Salud Publica. 2007;21(2):164-76.

14. Oliveira FB, Guedes HKA, Oliveira TBS, Lima Júnior JF. (Re) Construindo cenários de atuação em saúde mental na estratégia saúde da família. RBPS. 2011; 24(2):109-15.

15. Ferreira Filha MO, Dias MD, Andrade FB, Lima EAR, Ribeiro FF, Silva MSS. A terapia comunitária como estratégia de promoção à saúde mental: o caminho para o empoderamento Rev. Eletr. Enf. [Internet]. 2009;11(4) [acesso em 25 jun 2014]. Disponível: http:// www.fen.ufg.br/revista/v11/n4/v11n4a22.htm

16. Sucigan DHI, Toledo VP, Garcia APRF. Acolhimento e saúde mental: desafio profissional na estratégia saúde da família. Rev Rene. 2012;13(1):2-10.

17. Gonçalves RMDA, Pedrosa LAK. Perfil dos enfermeiros da estratégia saúde da família e suas habilidades para atuar na saúde mental. Cienc Cuid Saude. 2009;8(3):34551.

18. Waidman MAP, Marcon SS, Pandini A, Bessa JB, Paiano $M$. Assistência de enfermagem às pessoas com transtornos mentais e às famílias na atenção básica. Acta Paul Enferm. 2012; 25(3):346-51.

19. Moura MC, Bernardes SB, Rocha MLF. A percepção dos enfermeiros de uma universidade pública do Piauí sobre saúde mental na estratégia saúde da família. Enfermagem em Foco. 2010;1(2):66-8. saúde mental no programa de saúde da família. . Rev. bras. enferm. 2007;60(4):391-5

21. Caixeta CC, Moreno V. O enfermeiro e as ações de saúde mental nas unidades básicas de saúde. Rev. Eletr. Enf. [Internet]. 2008;10(1) [acesso em: 25 jun 2014]. Disponível: http://www.fen.ufg.br/revista/v10/ n1/v10n1a16.htm

22. Zanetti TG, Van Der Sand ICP, Girardon-Perlini NMO, Kopf AW, Abreu PB. Perfil socioprofissional e formação de profissionais de equipes de saúde da família: um estudo de caso. Cienc Cuid Saúde. 2010; 9(3):448-55.

23. Ribeiro LM, Medeiros SM, Albuquerque JS, Fernandes SMBA. Saúde mental e enfermagem na estratégia saúde da família: como estão atuando os enfermeiros? Rev. Esc. Enferm. USP. 2010; 44(2):376-82.

24. Oliveira FB, Costa e Silva JC, Silva VHF, Cartaxo CKA. O trabalho de enfermagem em saúde mental na estratégia de saúde da família. Rev Rene. 2011;12(2): 229-37.

25. Ministério da Saúde (BR), Secretaria de Atenção à Saúde. Cadernos de Atenção Básica, n. 34. Brasília: Ministério da Saúde; 2013.

26. Amarante AL, Lepre AS, Gomes JLD, Pereira AV, Dutra VFD. As estratégias dos enfermeiros para o cuidado em saúde mental no programa saúde da família. Texto Contexto Enferm. 2011; 20(1):85-93.

27. Pinto AGA, Jorge MSB, Vasconcelos MGF, Sampaio JJC, Lima GP, Bastos VC, et al. Apoio matricial como dispositivo do cuidado em saúde mental na atenção primária: olhares múltiplos e dispositivos para resolubilidade. Ciênc. saúde coletiva. 2012;17(3):65360. 